\title{
Software Defined Radio Provides New Opportunities for Hands-on RF Education
}

\author{
Erik Luther ${ }^{l}$, Julia Dinolfo ${ }^{l}$, and Sachin Katti ${ }^{2}$ \\ ${ }^{l}$ National Instruments, ${ }^{2}$ Stanford University \\ Erik.Luther@ni.com
}

\begin{abstract}
R F$ and communications topics are traditionally taught using a theoretical, math-focused approach where students derive formulas and build simulations. Many educators see the value of adding a lab component so students can apply the theory with prototype implementations and experiment with live signals. However, the opportunity to do so is limited by a lack of affordable, accessible, and relevant tools.

This paper describes how Stanford University enabled cutting edge, hands-on RF education in an undergraduate course. We share best practices for deploying a successful and affordable hands-on RF and communications lab using NI LabVIEW software and NI USRP radios. Students rated the class 4.94/5.0 - making it one of the highest ratings among all classes in the School of Engineering at Stanford University.
\end{abstract}

Keywords: RF communications, radio, technology, lab, experiment, equipment, teaching, learning, innovative.

\section{INTRODUCTION}

The fundamentals of wireless communications are quickly becoming an important tool needed across many design oriented disciplines. The over 1.5 billion mobile phones sold annually [1] make up only a fraction of the growing number of wireless devices in use. Rapidly advancing wireless technologies are allowing new research into areas that extend from agriculture, to biomedical, to more traditional electrical engineering disciplines. Examples include RFID tags being used as humidity monitors in farm soil [2], pill-sized wireless sensors for transmitting patient biometrics [3], and the development of next generation mobile communications devices that use less battery power and provide a faster and more reliable service [4]. As today's students graduate they will have opportunities to redefine how we use RF and wireless technologies to not only communicate but also monitor and interact with the world around us.

However, few students have a practical hands-on understanding of wireless communications systems upon graduation. As the world transitioned from analog to digital communications, equipment costs and rapidly changing technology has made building and maintaining a wireless communications lab challenging. The emergence of affordable software defined radio (SDR) platforms is providing new opportunities for hands-on wireless communication education.

In this paper we discuss the use of LabVIEW software to rapidly prototype communications systems designed around NI USRP software defined radio transceivers for both transmission and reception of RF signals across a wide range of frequencies. The platform provides an affordable laboratory solution with a lower learning curve that scales from teaching through to research. The low cost, open LabVIEW-based programming approach, and improved documentation have addressed many of the challenges documented by other educators using the USRP equipment to introduce wireless communications in an educational setting.[5],[6]

While many schools are at various stages of course integration, we focus on one of the first completed LabVIEW / USRP oriented RF courses piloted at Stanford University, EE 49 Building Networked Systems. Both the positive student feedback and the accomplishments of these students in a single quarter seem to indicate that this approach has merit and warrants further research across multiple institutions and demographics. Thus far, this learning approach appears to boost student engagement and better prepare them for both academic and professional careers in wireless communications.

\section{MAIN BODY}

Recognizing that undergraduate students have an interest in wireless communications, but few options for hands-on experimentation, Professor Katti of Stanford University introduced EE 49 Building Networked Systems in the spring quarter of 2011. The goal was to provide a holistic approach to digital communications, using the lectures to build intuition and the laboratory time to take deep dives into the implementation details (with a level of abstraction that ensured success). The course was opened for enrollment across CE, CS, and EE disciplines with the objective of attracting students as early as their second year of studies. Software defined radio allows the students to cover both basic and complex applications using a single platform.[7] 


\subsection{Course Flow}

Over the course of the 10 week quarter, students were challenged to build and explore each stage of a complete communications system from bits, to signals, and back again (see Fig 1). The course focused on baseband coding and modulation techniques where upconversion/ downconversion to/ from RF was implemented in the NI USRP hardware and the communications channel was an over-the-air communications channel.

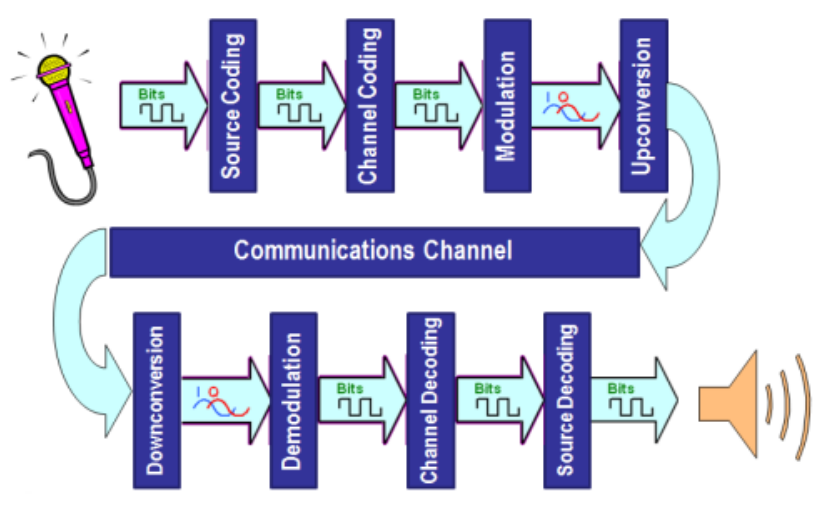

Fig. 1. Digital communications system

Beginning with basic concepts of Information Theory, students learned how messages become bits and why it is challenging to transmit and receive bits without errors, focusing on an AWGN (Additive White Gaussian Noise) channel. This was followed by practical challenges in receiving a digital message that might be distorted by an LTI (Linear Time Invariant) channel. Students were then introduced to the USRP SDR platform, and in the following labs used it to build a binary phase-shift keying (BPSK) radio. For example, in the fourth lab students studied the concept of demodulation and implemented parts of a wireless BPSK receiver for a USRP-to-USRP link. A single transmitter repeatedly transmitted a BPSK packet of information for the students to decode. Each student, with their own computer and NI USRP, developed a receiver to demodulate and decode the signal. The students implemented channel correction, a BPSK symbol de-mapper, a preamble detection scheme, packet decoding, and cyclic redundancy check (CRC) error detecting code over the course of the two-week lab project. The flow of labs allowed students to use the code they developed in a previous lab for packet decoding in the final lab to establish a wireless link. The course culminated in a project in which students built a system for wireless text messaging between two computers. Throughout the course they gained experience with channel coding, modulation, timing recovery, random bit generation, and many other topics associated with a modern digital communication system (refer to Table 1).
Table 1. EE 49 course outline

\begin{tabular}{|ll|}
\hline 1 & Source Coding \\
2 & Packet Communication, Sync, and Channel \\
& Correction \\
3 & Modulation \\
4 & Demodulation \\
5 & Design Challenge: Packet based Transceiver \\
\hline
\end{tabular}

It was clear from the students' first introduction to the USRP that simple examples of decoding live broadcast FM radio, examining the $850 \mathrm{MHz}$ GSM cell phone uplink, and demodulation of a wireless key fob created an emotional connection between classroom materials and their every-day lives. The students quickly realized the power of the tools and immediately began to brainstorm on how these simple examples work and how they could be extended to cover more complex applications. Students felt empowered by the platform and developed a stronger desire for knowledge that both the lecture materials and hands-on experiments were designed to fulfill.

\subsection{Software Defined Radio Platform}

The laboratory platform used for this course consisted of National Instruments LabVIEW software and NI USRP hardware, as seen in Fig. 2. Together, these tools served as a software defined radio platform for the course allowing students to rapidly prototype both communications and signal processing algorithms and then experiment with live over-the-air signals. LabVIEW system design software provided a graphical user interface and methods for graphically designing algorithms by combining both textual and graphical mathbased programming approaches. Support for $\mathrm{m}$-file script is provided through the LabVIEW MathScript module. Hardware control in LabVIEW is accomplished through the NI-USRP hardware driver. Running in the Windows operating system, students could design the software aspects of their software defined radio and control the hardware to make use of the wide frequency range from $50 \mathrm{MHz}$ to $2.2 \mathrm{GHz}$ with an instantaneous bandwidth of up to $20 \mathrm{MHz}$. This equipment allowed for the simple experiments done in the course as well as the ability to extend experiments into the bands used by other commercial standards such as broadcast FM radio, broadcast television, mobile phone bands, GPS, safety radio, and many others. 


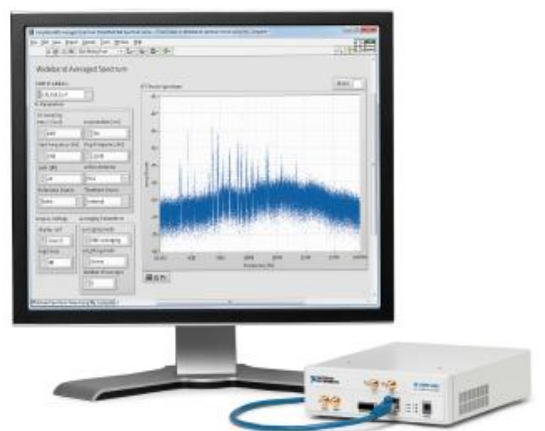

Fig. 2. NI USRP software defined radio

\subsection{Inside the USRP Hardware}

When connected to a host PC running LabVIEW the USRP becomes a software-defined radio. Incoming signals attached to the standard SMA connector are mixed down from RF using a direct-conversion receiver (DCR) to baseband I/Q components, which are sampled by a 2channel, $100 \mathrm{MS} / \mathrm{s}, 14-\mathrm{bit}$, analog-to-digital converter (ADC) as is illustrated in Fig 3. The digitized I/Q data follows parallel paths through a digital downconversion (DDC) process that mixes, filters, and decimates the input $100 \mathrm{MS} / \mathrm{s}$ signal to a user-specified rate. The downconverted samples are passed to the host computer at up to $20 \mathrm{MS} / \mathrm{s}$ over a standard Gigabit Ethernet connection. For transmission, baseband I/Q signal samples are synthesized by the host computer and fed to a USRP-292x at up to $20 \mathrm{MS} / \mathrm{s}$ over Gigabit Ethernet. The USRP hardware interpolates the incoming signal to 100 $\mathrm{MS} / \mathrm{s}$ using a digital upconversion (DUC) process and then converts the signal to analog with a dual-channel, 16bit, digital-to-analog converter (DAC). The resulting analog signal is then mixed up to the specified RF frequency.

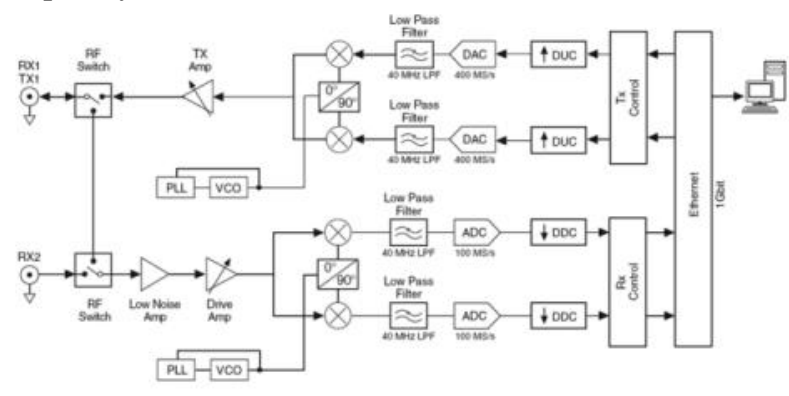

Fig. 3. USRP hardware configuration

\subsection{Student Reaction}

The response from students in the trial course was quite positive. Below is an expert of feedback provided by one of the students:

"It was incredible to actually see how the material we learned in classroom lectures is applied in practice in the lab," said Duarte. "I was not familiar with the USRP hardware prior to this course, but through understanding and using LabVIEW, I grew very familiar with programming the devices. LabVIEW itself was a pleasure to work with. It was very easy to get things working and debugging was quite simple. Overall, this class was easily my favorite in the three years I have spent at Stanford."

The students were able to easily view and interact with these processes by using the LabVIEW software and NI USRP driver. The LabVIEW development system is ideally suited for signal processing, programming tasks, and hardware interfacing with NI USRP software-defined radio hardware. Together, these tools enabled the students to develop and explore algorithms for processing received signals and synthesizing signals for transmission.

\section{RESULTS AND DISCUSSION}

While the original intent of this course was to stimulate interest in wireless communications for a broader base of undergraduates, the end of year course evaluations pointed to a much more profound result. Course evaluations affirmed that students were highly engaged in and benefitted greatly from the EE 49 class. Students rated the class 4.94/5.0, likely making it one of the highest ratings among all classes in the School of Engineering at Stanford.

Student comments echoed these strong ratings, with feedback such as: "Hands down the best EE class I've taken so far."

"Really great for a first-time class...the labs were really helpful for understanding concepts in practice and were interesting in design. I would recommend this [class] to all of my EE friends."

"Awesome class! I really enjoyed the lectures, and the labs were really cool because we got to use the hardware."

Many universities in North America have seen a decrease in ECE enrollments, and one reason commonly cited for this is our ability to give students relevant, hands-on lab experience. This solution helps to solve that issue in the area of wireless communications.

Because of the success in the trial course, Professor Katti has expanded course enrollment for the spring quarter of 2012 with up to 20 students and 20 lab stations. Katti 
anticipates this type of course will better prepare students for future signal processing and communications courses. Because of the accessibility of this educational solution, the platform is also applicable to a wide range of other introductory-level signal processing courses, such as information theory and signals and systems. Additionally, the platform scales with the students as they progress in their studies or move on to research or industry applications.

\section{CONCLUSIONS}

The positive nature of student feedback indicate that this teaching approach has merit and warrants further investigation across multiple institutions and demographics as a way to boost student engagement and better prepare them for both academic and professional careers in wireless communications. We believe that the approach taken at Stanford University can scale to other universities and enable similar student success. Wireless communications is a growing part of everyday life. LabVIEW software and the NI USRP hardware provide an affordable, accessible, and relevant solution for developing student proficiencies that will allow them to build interest and practical experience in the field. While the course is a work in progress, course materials are available as a reference for other universities interested in learning more about the approach. LabVIEW and the USRP are a viable method for introducing hands-on experiences in wireless communications and offer an alternative to simulation-only educational approaches.

\section{Acknowledgements}

I would like to recognize the EE 49 course graduate teaching assistants from spring 2011, Jeff Mehlman and Aditya Gudipati. As well as I would like to acknowledge the spring 2012 teacher's assistants, Stephen Hong and Vighnesh Rege. They worked hand-in-hand with Dr. Katti to develop the course materials and provided valuable guidance to the students throughout the course.

\section{References}

[1] Ramon Llamas, Worldwide Mobile Phone Market Maintains Its Growth Trajectory in the Fourth Quarter Despite Soft Demand for Feature Phones, According to IDC, 2011, Available as of Feb 1, 2011 from

http://www.idc.com/getdoc.jsp?containerId=prUS23297412

[2] Fu Gang Wang, Li Jia Xu, Chun Long Du, Yang Yang, Ye Sen Li, "The Design of Intelligent Water-Saving Irrigation System for Greenhouse," Engineering Applied Mechanics and Materials (Volumes 121 - 126), no. 6, pp. 4826-4831, 2011. Available as of May 2012 from

http://www.scientific.net/AMM.121-126.4826
[3] Guobing Pan1, Litong Wang, Swallowable Wireless Capsule Endoscopy: Progress and Technical Challenges, 2011, 9pp, Article ID 841691, Available as of May, 2012 from

http://downloads.hindawi.com/journals/grp/2012/841691. pdf

[4] Roman Marsalek, Demian Lekomtcev, "Testbed for Cognitive Radio Networks Based on USRP2/N200 Modules," in COCORA 2012, The Second International Conference on Advances in Cognitive Radio, 9 pp., 2012. Available as of May, 2012 from http://www.thinkmind.org/index.php?view=article\&articl eid=cocora 2012_2_40_60072

[5] Joseph Hoffbeck, "TEACHING COMMUNICATION SYSTEMS USING THE UNIVERSAL SOFTWARE RADIO PERIPHERAL (USRP) AND GNU RADIO," in American Society of Engineering Education Conference, 2009 Annual Conference \& Exposition, 9 pp., 2009.

[6] Thad B Welch, Sam Shearman, "LABVIEW, THE USRP, AND THEIR IMPLICATIONS ON SOFTWARE DEFINED RADIO," in American Society of Engineering Education Conference, 2011 Annual Conference \& Exposition, 13 pp., 2011.

[7] Sharlene Katz, James Flynn, "USING SOFTWARE DEFINED RADIO (SDR) TO DEMONSTRATE CONCEPTS IN COMMUNICATIONS AND SIGNAL PROCESSING COURSES," in American Society of Engineering Education Conference, 2009 Annual Conference \& Exposition, 19 pp., 2009. 